Document downloaded from:

http://hdl.handle.net/10251/161594

This paper must be cited as:

Bahrami, Z.; Keshtkar, A.; Ghorbani, A.; Esteban González, H. (2020). Efficient analysis of metal and dielectric rods in rectangular waveguide with ferrite magnetized transversely. Journal of Electromagnetic Waves and Applications. 34(2):141-153. https://doi.org/10.1080/09205071.2019.1690589

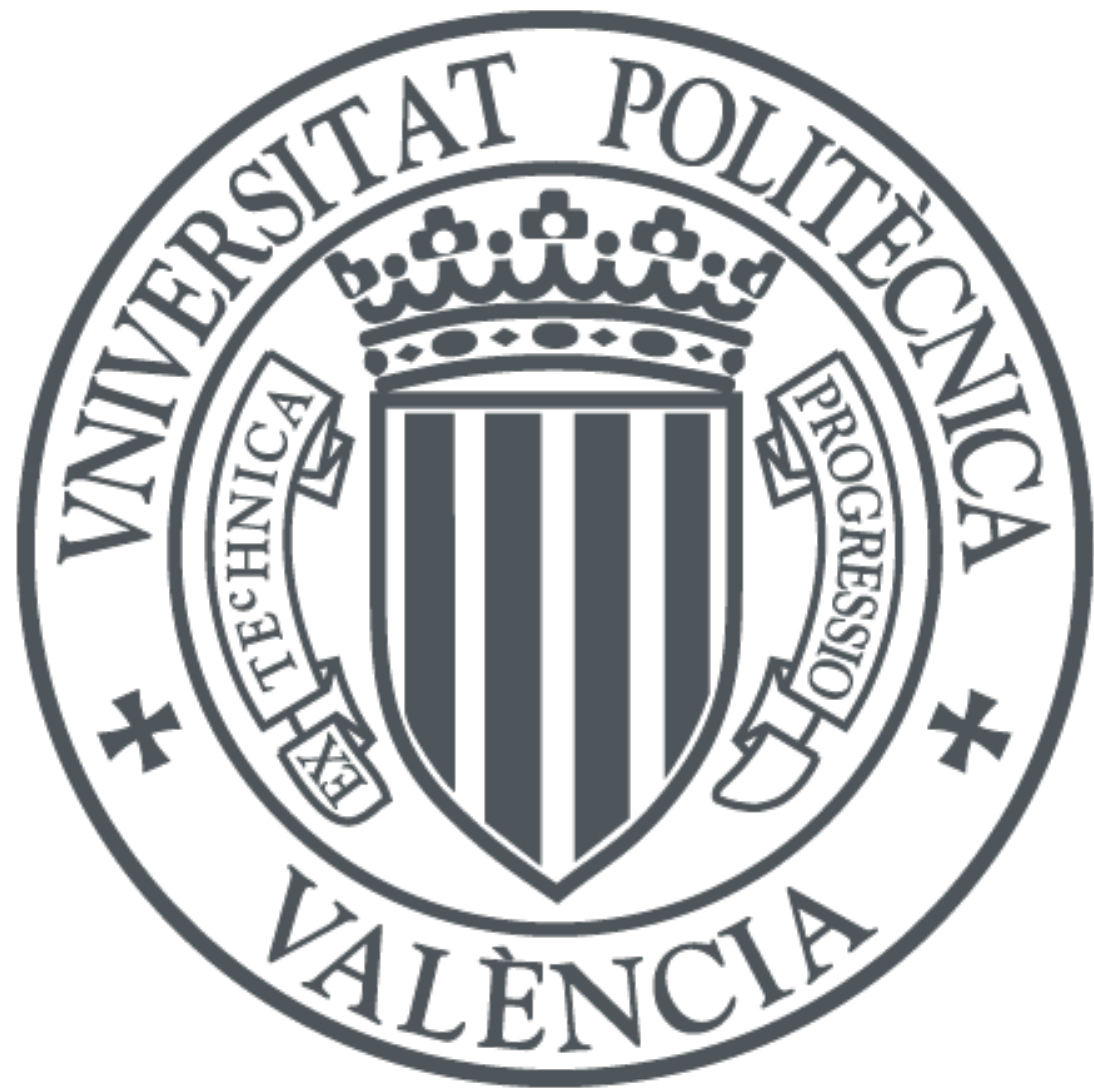

The final publication is available at

https://doi.org/10.1080/09205071.2019.1690589

Copyright Taylor \& Francis

Additional Information

This is an Author's Accepted Manuscript of an article published in Zahra Bahrami, Asghar Keshtkar, Ayaz Ghorbani \& Hector Esteban (2020) Efficient analysis of metal and dielectric rods in rectangular waveguide with ferrite magnetized transversely, Journal of Electromagnetic Waves and Applications, 34:2, 141-153 [copyright Taylor \& Francis], available online at: http://www.tandfonline.com/10.1080/09205071.2019.1690589 


\section{Efficient analysis of metal and dielectric rods in rectangular waveguide with ferrite magnetized transversely}

Zahra Bahrami,

Asghar Keshtkar,

Ayaz Ghorbani and

Hector Esteban

\section{ABSTRACT}

This paper presents a new analytical method based on mode matching technique for a waveguide full of anisotropic material with rods. It is proved that mode matching technique is applicable for the waveguide with anisotropic materials. The problem is considered as a $2 \mathrm{D}$ electromagnetic problem assuming no field variation normal to the waveguide length. Incident and scattered fields from circular cylinder rods in ferrite medium are expanded with cylindrical modes, and the fields in the waveguide ports are expanded using progressive and regressive modal summations. In both regions, extraordinary wave number is considered for the fields. Finally, the hybrid mode matching between guided and cylindrical modes is done by projecting continuity equations in the circular boundary containing the rod. The fast Fourier transform is used to solve the matching in the presented method. It is shown, that the new method runs faster than commercial software packages, with very good accuracy. 


\section{Introduction}

Consider a cascade connection of multiple Two-port microwave device, including several sections as empty waveguides, discontinuities, waveguides loaded with the dielectric or metallic rods, waveguides loaded with anisotropic materials, etc. In the analysis of such as these devices, Generalized Scattering Matrix (GSM) of each section must be connected to each other to obtain a total GSM [1-5]. The empty waveguide scattering matrix can be obtained analytically [2]. The discontinuities can be analysed using a modal method [6]. The waveguide loaded with any shape obstacles can be analysed using a variational method [7], finite element method (FEM) [8], finite and boundary element method [9,10] and resonant method [11]. Authors in [12], analysed H-plane waveguides loaded with a circular metal or dielectric rods, using an efficient method based on a hybrid mode matching technique. In [12], the fast Fourier transform (FFT) was used to solve matching between cylindrical and guided modes, efficiently. The presented method in [12] increased accuracy and stability of the analysis procedure in compare to other methods such as FEM or finite difference time domain (FDTD) method. Authors in [13], analysed substrate integrated waveguides (SIW) devices with multiple accessing ports, using the method described in [12], efficiently. In fact, in this paper each via of the SIW was considered as a metal rod, and scattering from each of them was considered in the total GSM. The progress of [13] in compare to [12] is the consideration of electromagnetic coupling between vias and consideration of multiple ports for the SIW.

In other hand, ferrimagnetic compounds have high resistivity and a significant amount of anisotropy at microwave frequencies. They are used in waveguides for construction of passive devices such as filters, isolators or phase shifters predominantly [2]. Using circular rods in geometry of these devices is unavoidable. Until now, all the efforts in analysis of waveguides loaded by the metallic or dielectric rods were done in such a way that the waveguide is filled with isotropic materials. But, it is possible that the waveguide full of anisotropic materials (such as a ferrite) be loaded by metallic or dielectric rods. For example, consider a SIW filled with ferrite magnetized transversely or longitudinally, which the structure can be used for ferrite phase shifter construction, antenna or filters [14-17]. Each via in this SIW structure can be considered as metal cylindrical rod. So, in the GSM of this structure, scattering from each via should be considered reaching a good accuracy and stability.

In this paper, the authors generalize the method presented in [12] in the anisotropic material. In fact, it will be examined that the hybrid mode matching technique can be used for an anisotropic material or not. Finally, total GSM for this structure will be obtained, and the analytical results will be verified with commercial software packages.

\section{Formulation}

The problem is divided in two regions, as it is depicted in Fig.1. The region "B" that encloses the circular cylindrical rod, is considered as a ferrite medium. The fields in this region, are expanded in a series of cylindrical modes with an extraordinary wave number. The region " $\mathrm{A}$ " is considered as a waveguide full of ferrite magnetized transversely. So, the fields in this region are in a series of progressive and regressive guided modes summations with the extraordinary wave number. In the follow, each region is considered separately, in order to extracting electromagnetic fields.

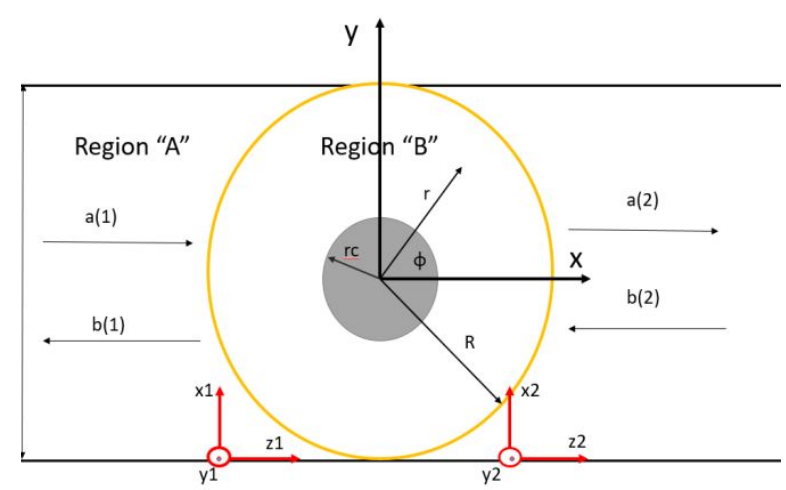

Fig. 1. Problem Geometry 


\subsection{Electromagnetic Fields in the Region " $B$ "}

According to the Global coordinate shown in Fig.1, the region "B" is filled by ferrite magnetized in the $\mathrm{z}$ direction. So, the permeability tensor in this region becomes [2]:

$$
\bar{\mu}=\left[\begin{array}{ccc}
\mu & j \kappa & 0 \\
-j \kappa & \mu & 0 \\
0 & 0 & \mu_{0}
\end{array}\right]
$$

Where $\mu_{0}$ is the permeability of free space, and $\mu$ and $\kappa$ are:

$$
\begin{aligned}
& \mu=\mu_{0}\left(1+\frac{\omega_{0} \omega_{m}\left(\omega_{0}^{2}-\omega^{2}\right)+\omega_{0} \omega_{m} \omega^{2} \alpha^{2}}{\left[\omega_{0}^{2}-\omega^{2}\left(1+\alpha^{2}\right)\right]^{2}+4 \omega_{0}^{2} \omega^{2} \alpha^{2}}-j \frac{\alpha \omega \omega_{m}\left[\omega_{0}^{2}+\omega^{2}\left(1+\alpha^{2}\right)\right]}{\left[\omega_{0}^{2}-\omega^{2}\left(1+\alpha^{2}\right)\right]^{2}+4 \omega_{0}^{2} \omega^{2} \alpha^{2}}\right) \\
& \kappa=j \mu_{0}\left(\frac{-\omega \omega_{m}\left[\omega_{0}^{2}-\omega^{2}\left(1+\alpha^{2}\right)\right]}{\left[\omega_{0}^{2}-\omega^{2}\left(1+\alpha^{2}\right)\right]^{2}+4 \omega_{0}^{2} \omega^{2} \alpha^{2}}+j \frac{2 \omega_{0} \omega_{m} \omega^{2} \alpha}{\left[\omega_{0}^{2}-\omega^{2}\left(1+\alpha^{2}\right)\right]^{2}+4 \omega_{0}^{2} \omega^{2} \alpha^{2}}\right)
\end{aligned}
$$

Here $\omega$ is the angular frequency, $\omega_{0}$ is the precession frequency or Larmor frequency $\left(\omega_{0}=\mu 0 \gamma \mathrm{H} 0\right), \mathrm{H}_{0}$ is the internal dc magnetic field, $\mathrm{M}_{\mathrm{s}}$ is the saturation magnetization, $\Delta \mathrm{H}$ is the resonance linewidth, $\gamma$ is the gyromagnetic ratio $\left(\gamma=1.75 \times 10^{11} \mathrm{C} / \mathrm{Kg}\right), \alpha$ is the damping factor $\left(\alpha=\mu_{0} \gamma \Delta \mathrm{H} / \Delta \omega_{0}\right)$, and $\omega_{\mathrm{m}}$ is $\mu_{0} \gamma \mathrm{M}_{\mathrm{s}}$.

Now, the incident and scattered fields from circular cylindrical rod, are written in series of cylindrical modes [2]:

$$
\begin{aligned}
& \stackrel{\mathrm{r}}{E^{i n}}(\rho, \varphi)=\hat{z} \sum_{n=-N_{i}}^{N_{i}} i_{n} J_{n}\left(k_{f} \rho\right) e^{j n \varphi} \\
& \stackrel{\mathrm{r}}{\mathrm{r}}^{s c}(\rho, \varphi)=\hat{z} \sum_{q=-N_{s c}}^{N_{s c}} c_{q} H_{q}^{(2)}\left(k_{f} \rho\right) e^{j q \varphi}
\end{aligned}
$$

Where the coefficients, $i_{n}$ and $c_{q}$, are the incident and scattered field spectra in region "B", respectively, and $N_{i}$ and $N_{s c}$ are the truncation indexes for the summation of incident and scattered cylindrical modes, respectively. Both numbers must be high enough to ensure good accuracy of the results [12]. The $k_{f}=\omega \sqrt{\varepsilon \mu_{e f f}}$ is the extraordinary wave number in ferrite media, which $\varepsilon$ and $\mu_{e f f}=\left(\mu^{2}-K^{2}\right) / \mu$ are permittivity and effective permeability of the ferrite media, respectively.

The scattered field spectra, can be related to the incident field spectra, as [18]:

$$
c_{q}=\sum_{q=-N_{i}}^{N_{i}} d_{q n} i_{n}
$$

Where $d_{q n}$ is the element of the scattering matrix, D, for the rod. For a centered circular rod, D becomes a diagonal matrix and we have:

$c_{q}=d_{n n} i_{n}$

For a metallic centered circular rod: 


$$
d_{n n}=-\frac{J_{n}\left(k_{f} r_{c}\right)}{H_{q}^{(2)}\left(k_{f} r_{c}\right)}
$$

And for a dielectric centered circular rod:

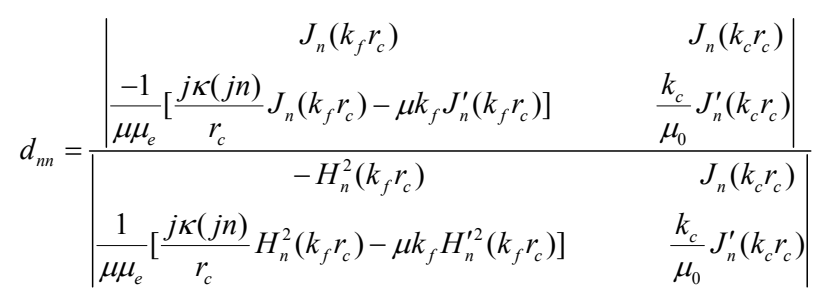

Where $\mathrm{r}_{\mathrm{c}}$ and $k_{c}=\omega \sqrt{\varepsilon_{c} \mu_{c}}$ are radius of the circular rod and wave number in dielectric media by permittivity and permittivity equal to $\varepsilon_{\mathrm{c}}$ and $\mu_{\mathrm{c}}$, respectively.

Finally, the total electric field in this region for a centered cylindrical rod, can be written as the summation of incident and scattered electric fields:

$$
\stackrel{\mathrm{r}}{E}(\rho, \varphi)=\hat{z} \sum_{n=-N_{i}}^{N_{i}}\left(J_{n}\left(k_{f} \rho\right)+d_{n n} H_{n}^{(2)}\left(k_{f} \rho\right)\right) i_{n} e^{j n \varphi}
$$

Now, using Maxwell's curl equations, the components of magnetic field in this region become:

$$
\begin{aligned}
& H_{\rho}=\frac{-1}{j \omega \mu \mu_{e}}\left(\frac{\mu}{\rho} \sum_{n=-N_{i}}^{N_{i}} j n\left(J_{n}\left(k_{f} \rho\right)+d_{n n} H_{n}^{(2)}\left(k_{f} \rho\right)\right) i_{n} e^{j n \varphi}+j \kappa \sum_{n=-N_{i}}^{N_{i}} k_{f}\left(J_{n}^{\prime}\left(k_{f} \rho\right)+d_{n n} H_{n}^{(2)^{\prime}}\left(k_{f} \rho\right)\right) i_{n} e^{j n \varphi}\right) \\
& H_{\varphi}=\frac{-1}{j \omega \mu \mu_{e}}\left(\frac{j \kappa}{\rho} \sum_{n=-N_{i}}^{N_{i}} j n\left(J_{n}\left(k_{f} \rho\right)+d_{n n} H_{n}^{(2)}\left(k_{f} \rho\right)\right) i_{n} e^{j n \varphi}-\mu \sum_{n=-N_{i}}^{N_{i}} k_{f}\left(J_{n}^{\prime}\left(k_{f} \rho\right)+d_{n n} H_{n}^{(2)^{\prime}}\left(k_{f} \rho\right)\right) i_{n} e^{j n \varphi}\right)
\end{aligned}
$$

\subsection{Electromagnetic Fields in the Region " $A$ "}

In this region, local coordinate should be considered in electromagnetic fields. As it is depicted in Fig.1, the applied biased field is in y direction according to the local coordinate. So, the permeability tensor becomes [2]:

$$
\bar{\mu}=\left[\begin{array}{ccc}
\mu & 0 & -j \kappa \\
0 & \mu_{0} & 0 \\
j \kappa & 0 & \mu
\end{array}\right]
$$

In (13), $\mu$ and $\kappa$ are defined in (2) and (3), already.

Since both the geometry and excitation are invariant in height, only the family of the $\mathrm{TE}_{\mathrm{m} 0}$ modes are considered [12]. The tangential electromagnetic fields in this region, according to progressive and regressive guided modes, can be written as:

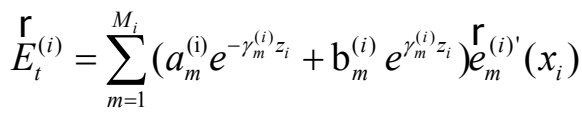




$$
{\stackrel{\mathrm{r}}{H_{t}^{(i)}}}^{(i)} \frac{1}{j \omega \mu \mu_{e}}\left(\sum_{m=1}^{M_{i}}\left(\mu \gamma a_{m}^{(\mathrm{i})} e^{-\gamma_{m}^{(i)} z_{i}}-\mu \gamma \mathrm{b}_{m}^{(i)} e^{\gamma_{m}^{(i)} z_{i}}\right) \mathbf{c}_{m}^{(i)^{\prime}}\left(x_{i}\right)+j \kappa \sum_{m=1}^{M_{i}}\left(a_{m}^{(\mathrm{i})} e^{-\gamma_{m}^{(i)} z_{i}}+\mathrm{b}_{m}^{(i)} e^{\gamma_{m}^{(i)} z_{i}}\right) \stackrel{\mathbf{r}_{m}^{(i) '}}{\left.\left(x_{i}\right)\right)}\right.
$$

Or in other word:

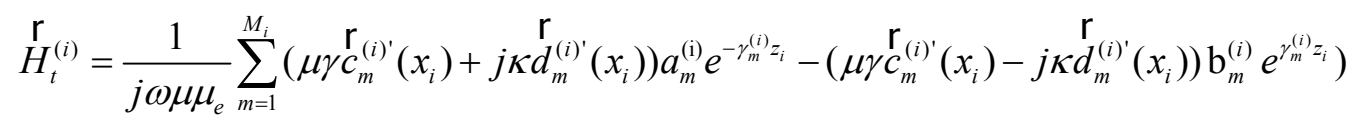

Where $i=1$ and $i=2$ are for the input and output ports, respectively, and:

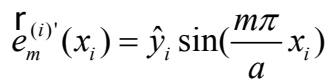

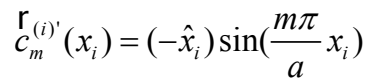

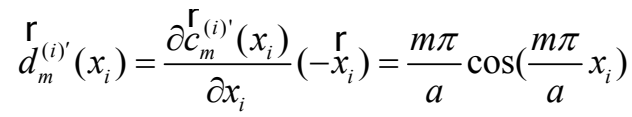

$$
\begin{aligned}
& \gamma_{(m)}^{(i)}=\sqrt{\left(\frac{m \pi}{a}\right)^{2}-k_{f}^{2}}
\end{aligned}
$$

In these equations [12]:

- $\quad m$ is the index of the correspondant guided mode.

- $\quad M_{i}, a_{i}$ and $b_{i}$ are respectively, the number of guided modes, the width and the height of the input $(i=1)$ and output $(i=2)$ ports. In the structures considered in this paper, $M_{2}=M_{1}, a_{2}=a_{1}$ and $b_{2}=b_{1}$.

- $\quad a_{m}^{(i)}$ and $b_{m}^{(i)}$ are, respectively the amplitude of the waves in the input $(i=1)$ and output $(i=2)$ ports.

- $\quad x_{i}$ and $z_{i}$ are the coordinates local to the input $(i=1)$ and output $(i=2)$ ports.

The amplitude of the guided waves and wave propagation constant can be referenced in a more compact way defining the following vectors $\mathbf{a}, \mathbf{b}$ and $\gamma$ :

$$
a=\left[\begin{array}{l}
a^{(1)} \\
b^{(2)}
\end{array}\right], b=\left[\begin{array}{l}
b^{(1)} \\
a^{(2)}
\end{array}\right], \gamma=\left[\begin{array}{c}
-\gamma^{(1)} \\
\gamma^{(2)}
\end{array}\right]
$$

Where

$$
\begin{aligned}
& a^{(i)}=\left[a_{1}^{(i)}, \ldots, a_{M_{i}}^{(i)}\right]^{T}, i \in[1,2] \\
& b^{(i)}=\left[b_{1}^{(i)}, \ldots, b_{M_{i}}^{(i)}\right]^{T}, i \in[1,2] \\
& \gamma^{(i)}=\left[\gamma_{1}^{(i)}, \ldots, \gamma_{M_{i}}^{(i)}\right]^{T}, i \in[1,2]
\end{aligned}
$$

Now, using the defined vectors, the tangential fields outside the boundary, can be expressed in a compact way:

$$
\stackrel{\mathrm{r}}{E_{t}}(\varphi)=\hat{z} \sum_{n=1}^{M}\left[a_{n} e_{n}^{+}(\varphi)+\mathrm{b}_{n} e_{n}^{-}(\varphi)\right] g_{n}(\varphi)
$$




$$
\stackrel{\mathrm{r}}{H_{t}^{(i)}}=\hat{y} \frac{1}{j \omega \mu \mu_{e}}\left[\sum_{n=1}^{M}\left(\mu \gamma g_{n}(\varphi)+j \kappa f_{n}(\varphi)\right) a_{n} e_{n}^{+}(\varphi)-\sum_{n=1}^{M}\left(\mu \gamma g_{n}(\varphi)-j \kappa f_{n}(\varphi)\right) \mathrm{b}_{n} e_{n}^{-}(\varphi)\right]
$$

Where $\mathrm{M}=\mathrm{M}_{1}+\mathrm{M}_{2}$, and:

$$
\begin{aligned}
& e_{n}^{ \pm}(\varphi)= \begin{cases}e^{\mathrm{m} / \gamma_{n}^{(1)} z_{1}(\varphi)}, & \varphi \in\left[\frac{\pi}{2}, \frac{3 \pi}{2}\right], n \leq M_{1} \\
e^{ \pm \gamma_{n-M_{1} z_{2}^{(2)}(\varphi)}}, & \varphi \in\left[-\frac{\pi}{2}, \frac{\pi}{2}\right], n \mathrm{f} M_{1} \\
0 & \text { other }\end{cases} \\
& g_{n}(\varphi)= \begin{cases}e_{n}^{(1)^{\prime}}\left(x_{1}(\varphi)\right), & \varphi \in\left[\frac{\pi}{2}, \frac{3 \pi}{2}\right], n \leq M_{1} \\
e_{n-M 1}^{(2)}\left(x_{2}(\varphi)\right), & \varphi \in\left[-\frac{\pi}{2}, \frac{\pi}{2}\right], n \mathrm{f} M_{1} \\
0 & \text { other }\end{cases} \\
& f_{n}(\varphi)= \begin{cases}d_{n}^{(1)^{\prime}}\left(x_{1}(\varphi)\right), & \varphi \in\left[\frac{\pi}{2}, \frac{3 \pi}{2}\right], n \leq M_{1} \\
d_{n-M 1}^{(2)}\left(x_{2}(\varphi)\right), & \varphi \in\left[-\frac{\pi}{2}, \frac{\pi}{2}\right], n \mathrm{f} M_{1} \\
0 & \text { other }\end{cases}
\end{aligned}
$$

\subsection{Mode Matching Procedure}

Now, mode matching is solved by projecting the equations resulting from enforcing field continuity in circular boundary $\rho=R$, to the modes of region $A$ and $B$.

Enforcing the tangential fields on the circular boundary between region A and B gives:

$$
\begin{aligned}
& \sum_{n=-N_{i}}^{N_{i}} A_{n} e^{j n \varphi} i_{n}=\sum_{n=1}^{M}\left(a_{n} e_{n}^{+}(\varphi)+b_{n} e_{n}^{-}(\varphi)\right) g_{n}(\varphi) \\
& \sum_{n=-N_{i}}^{N_{i}}\left(B_{n}(\hat{\rho} \cdot \hat{y})+C_{n}(\hat{\varphi} \cdot \hat{y})\right) e^{j n \varphi} i_{n}=\frac{1}{j \omega \mu \mu_{e}}\left[\sum_{n=1}^{M}\left(\mu \gamma g_{n}(\varphi)+j \kappa f_{n}(\varphi)\right) a_{n} e_{n}^{+}(\varphi)-\sum_{n=1}^{M}\left(\mu \gamma g_{n}(\varphi)-j \kappa f_{n}(\varphi)\right) \mathrm{b}_{n} e_{n}^{-}(\varphi)\right]
\end{aligned}
$$

Where

$$
\begin{aligned}
& A_{n}=J_{n}\left(k_{f} R\right)+d_{n n} H_{n}^{(2)}\left(k_{f} R\right) \\
& B_{n}=\frac{-1}{j \omega \mu \mu_{e}}\left(\frac{j \mu}{R}\left(n\left(J_{n}\left(k_{f} R\right)+d_{n n} H_{n}^{(2)}\left(k_{f} R\right)\right)\right)+j \kappa\left(k_{f}\left(J_{n}^{\prime}\left(k_{f} R\right)+d_{n n} H_{n}^{(2))^{\prime}}\left(k_{f} R\right)\right)\right)\right)
\end{aligned}
$$




$$
C_{n}=\frac{-1}{j \omega \mu \mu_{e}}\left(\frac{j \kappa}{R}\left(j n\left(J_{n}\left(k_{f} R\right)+d_{n n} H_{n}^{(2)}\left(k_{f} R\right)\right)\right)-\mu\left(k_{f}\left(J_{n}^{\prime}\left(k_{f} R\right)+d_{n n} H_{n}^{(2)^{\prime}}\left(k_{f} R\right)\right)\right)\right)
$$

Projecting over the inner modes of region $\mathrm{B}, e^{-j m \varphi}$, in both side of the equality, we obtain:

$$
\begin{aligned}
& \sum_{n=-N_{i}}^{N_{i}} I_{m n} i_{n}=\sum_{n=1}^{M}\left(J_{m n} a_{n}+K_{m n} \mathrm{~b}_{n}\right) \\
& \sum_{n=-N_{i}}^{N_{i}} L_{m n} i_{n}=\sum_{n=1}^{M}\left(M_{m n} a_{n}-N_{m n} \mathrm{~b}_{n}\right)
\end{aligned}
$$

Where $m \in\left[-N_{i}, N_{i}\right]$, and:

$$
\begin{aligned}
& I_{m n}=\int_{0}^{2 \pi} A_{n} e^{j n \varphi} e^{-j m \varphi} d \varphi \\
& L_{m n}=\int_{0}^{2 \pi}\left(B_{n} \sin \varphi+C_{n} \cos \varphi\right) e^{j n \varphi} e^{-j m \varphi} d \varphi \\
& J_{m n}=\int_{0}^{2 \pi} g_{n}(\varphi) e_{n}^{+}(\varphi) e^{-j m \varphi} d \varphi \\
& K_{m n}=\int_{0}^{2 \pi} g_{n}(\varphi) e_{n}^{-}(\varphi) e^{-j m \varphi} d \varphi
\end{aligned}
$$

Projecting (30) and (31) to the modes of the outer region, $g_{m}^{*}$, gives:

$$
\begin{aligned}
& \sum_{n=-N_{i}}^{N_{i}} O_{m n} i_{n}=\sum_{n=1}^{M}\left(P_{m n} a_{n}+Q_{m n} \mathrm{~b}_{n}\right) \\
& \sum_{n=-N_{i}}^{N_{i}} V_{m n} i_{n}=\sum_{n=1}^{M}\left(U_{m n} a_{n}-T_{m n} \mathrm{~b}_{n}\right)
\end{aligned}
$$

Where $m \in[1, M]$, and:

$$
\begin{aligned}
& O_{m n}=\int_{0}^{2 \pi} A_{n} e^{j n \varphi} g_{m}^{*} d \varphi \\
& V_{m n}=\int_{0}^{2 \pi}\left(B_{n} \sin \varphi+C_{n} \cos \varphi\right) e^{j n \varphi} g_{m}^{*} d \varphi \\
& P_{m n}=\int_{0}^{2 \pi} g_{n}(\varphi) e_{n}^{+}(\varphi) g_{m}^{*} d \varphi
\end{aligned}
$$




$$
\begin{aligned}
& Q_{m n}=\int_{0}^{2 \pi} g_{n}(\varphi) e_{n}^{-}(\varphi) g_{m}^{*} d \varphi \\
& U_{m n}=\int_{0}^{2 \pi} \frac{1}{j \omega \mu \mu_{e}}\left(\mu \gamma g_{n}(\varphi)+j \kappa f_{n}(\varphi)\right) e_{n}^{+}(\varphi) g_{m}^{*} d \varphi \\
& T_{m n}=\int_{0}^{2 \pi} \frac{1}{j \omega \mu \mu_{e}}\left(\mu \gamma g_{n}(\varphi)-j \kappa f_{n}(\varphi)\right) e_{n}^{-}(\varphi) g_{m}^{*} d \varphi
\end{aligned}
$$

The GSM is obtained as [12]:

$$
S=\left(T+V I^{-1} K\right)^{-1}\left(U-V I^{-1} J\right)
$$

So the integrals I, V, T, K and J should be obtained which they can be solve analytically or by using the fast Fourier Transform (FFT) [12].

\subsection{Integral solution}

$I_{m n}$ can be solved analytically:

$$
I_{m n}= \begin{cases}A_{n} 2 \pi & m=n \\ 0 & m \neq n\end{cases}
$$

Remaining integrals which are used in GSM, can be solved using the (FFT) [12]:

$$
\begin{aligned}
& J_{m n} \approx Y^{9 / n}[-m] 2 \pi \\
& K_{m n} \approx \mathrm{Q}^{(n)}[-m] 2 \pi \\
& V_{m n} \approx \pi\left[C_{n}-j B_{n}\right] \%^{\% / m}[n+1]+\pi\left[C_{n}+j B_{n}\right] \%^{\% / m}[n-1]
\end{aligned}
$$

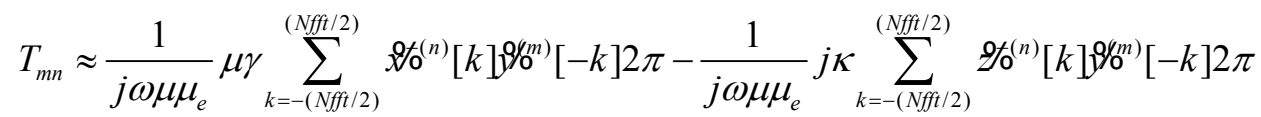

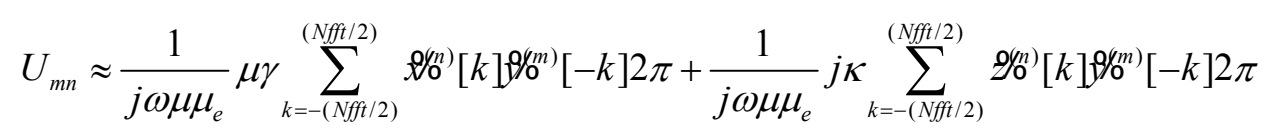

Where

$$
\begin{aligned}
& \left.\not \delta^{n}\right)[m]=F F T^{-1}\left\{e_{n}^{+}(\varphi) g_{n}(\varphi)\right\} \\
& \not \mathscr{\delta}^{(n)}[m]=F F T^{-1}\left\{e_{n}^{-}(\varphi) g_{n}(\varphi)\right\}
\end{aligned}
$$




$$
\begin{aligned}
& 9 \%^{(m)}[n]=F F T^{-1}\left\{g_{m}^{*}(\varphi)\right\} \\
& 20^{(n)}[m]=F F T^{-1}\left\{e_{n}^{+}(\varphi) f_{n}(\varphi)\right\} \\
& 20^{(n)}[m]=F F T^{-1}\left\{e_{n}^{-}(\varphi) f_{n}(\varphi)\right\}
\end{aligned}
$$

For multiple post, the same procedure as in [12] can be done, by using non diagonal scattering matrix. Another structure that can be solved using the analytical results of this paper and results in [13], is a SIW structures with ferrite substrate by multiple ports.

\section{Results}

In order to test the convergence of the presented method and choose the adequate values for the truncation indexes used in computational reasons in this paper, a similar convergence study as in [12] has been done with each one of these indexes. It shows, that the adequate values in this new formulation of hybrid mode matching is same as the previous values in [12]. The values are, the number of mode of the incident field spectrum, $N_{i}=P_{N_{i}} k_{f} R,\left(P_{N_{i m}}\right.$ =15), the number of point for the FFT, $N_{f f t}=2 \operatorname{ceil}\left(N_{i}\left(1+P_{N f f t}\right)\right)+1,\left(P_{N f f t}=2\right)$, and the number of the scattered field spectrum $N_{s c} \geq k_{f} r_{c}$.

We have tested the efficiency and accuracy of our method by analysing several cases. The results of this analysis will be compared with HFSS, because there isn't any analytical analysis in this field, already.

Before analysing our method and verifying the results, some aspects of ferrite material should be mentioned. In Fig.2 the effective permeability of the ferrite for different applied bias field (Ho), different magnetic saturation (Ms) and different frequencies is shown. As it is evident, the effective permeability becomes negative, in some regions. So, the ferrite material acts same as Metamaterials and the wave propagation becomes negative and the power flow become inversed, in these values. So the feature of ferrite should be considered in designing procedure.

In Fig.3 and Fi.g.4, the magnitude of reflection and transmission coefficients for a centered cylindrical PEC and a centered cylindrical dielectric in a waveguide full of ferrite magnetized transversely, is depicted, respectively, and the results are compared from our analysis and simulation with HFSS. The good agreements between simulation and analysis results is evident. But the total time for our analysis is 4 second, and the total time for HFSS is 3 minutes. This method reduces the computational time by $98 \%$ comparing it with HFSS temporal costs.

In fig.5, the magnitude of reflection and transmission coefficients for a constant diameter centered three circular cylinder metal post in a rectangular waveguide full of ferrite is depicted. Good agreement between simulation and analytical results is investigated

\section{Conclusion}

A new hybrid mode matching for the analysis of a single or multiple circular cylindrical rod in the rectangular waveguide full of ferrite magnetized transversely has been presented. It is proved in this paper that mode matching technique work very well for the anisotropic materials same as isotropic materials. The presented method reduces the computation time by approximately $98 \%$. Simulation results has been verified the analytical results very well. 


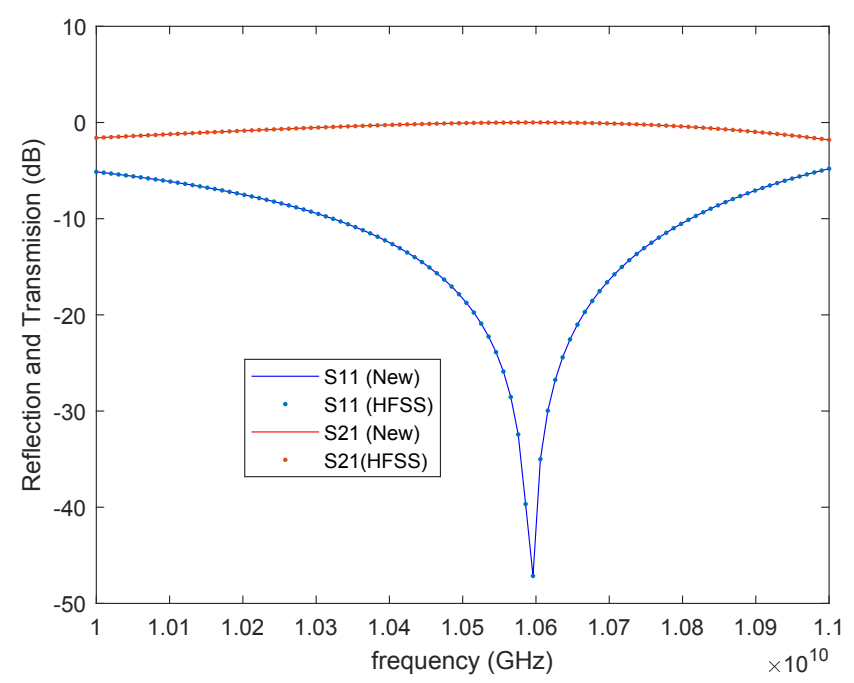

Fig. 3. Single centered metal post. $a=7.112 \mathrm{~mm}, r_{c}=0.07 a, H 0=3570$ Oe, $\Delta H=75$ Oe, $M s=1800$ Gauss, ferrite permittivity constant $\varepsilon_{r f}=13$. 


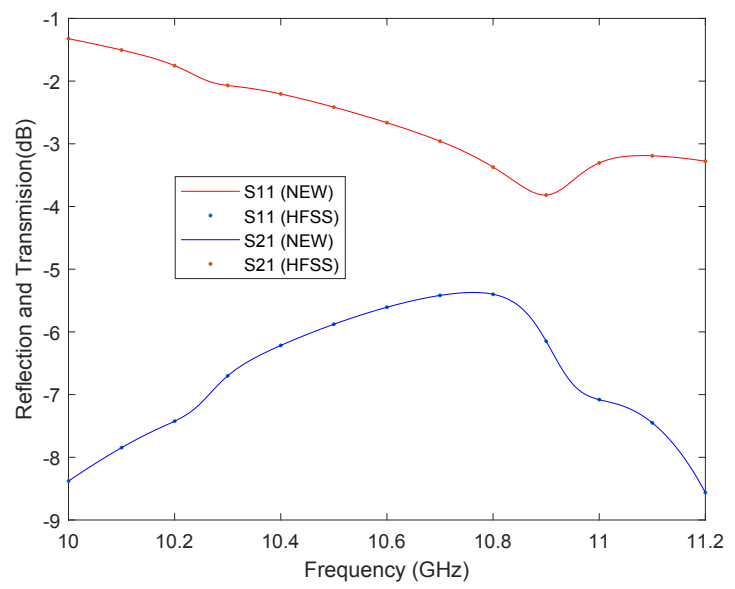

Fig. 4. Single centered dielectric post. $a=7.112 \mathrm{~mm}, r_{c}=0.07 a, H 0=3570 \mathrm{Oe}, \Delta H=75 \mathrm{Oe}$, $M s=1800$ Gauss, ferrite permittivity constant $\varepsilon_{r f}=13$ and dielectric permittivity constant $\varepsilon_{r}=24$.

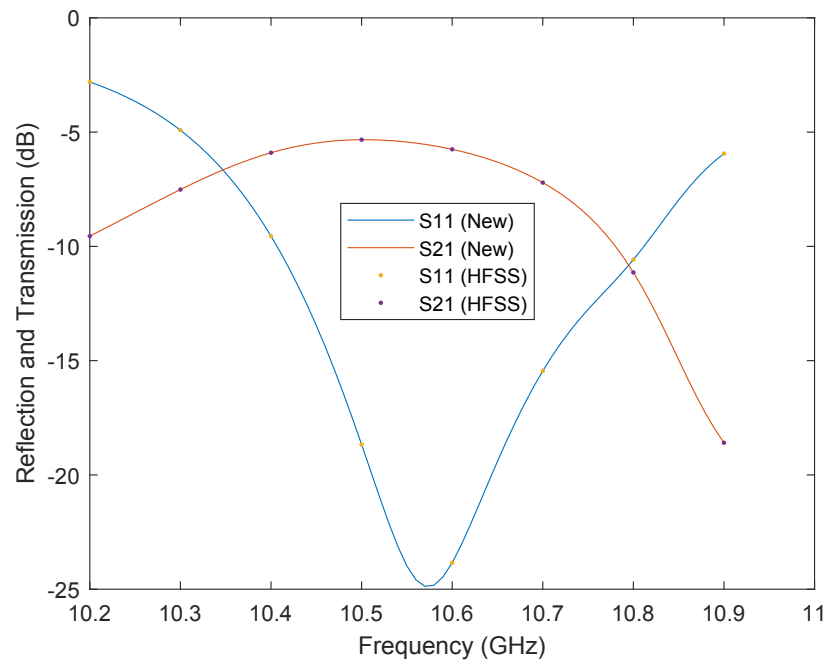

Fig. 5. Three centered metal post. $a=7.112 \mathrm{~mm}, r_{c}=0.07 a, H 0=3570 \mathrm{Oe}, \Delta H=75 \mathrm{Oe}, \mathrm{Ms}=1800$ Gauss, ferrite permittivity constant $\varepsilon_{r f}=13$.

\section{References}

[1] Bachiller, C., Gonzalez, H. E., Boria Esbert, V. E., Belenguer Martinez, A., Morro, J. V.: 'Efficient technique for the cascade connection of multiple Two-Port Scattering Matrices', IEEE trans. Microw. Theory tech., 2007, 55, (9), pp. $1880-1886$

[2] Pozar, D. M.: 'Microwave Engineering' (John Wiley \& Sons, New York, 2005)

[3] Marcuwitz, N.: 'Waveguide Handbook', (McGraw-Hill, New York, 1951)

[4] Lewin, L.: 'Advanced Theory of Waveguides', (McGraw-Hill, London, U.K, 1951) 
[5] Harrington, R.: 'Time-Harmonic Electromagnetic Fields', (McGrawHill, New York, 1961)

[6] Conciauro, G., Guglielmi, M., Sorrentino, M.: 'Advanced modal analysis - CAD techniques for waveguide components and filters', (Wiley, Chichester, 2000)

[7] Marcuwitz, N.: 'Waveguide Handbook', (McGraw-Hill, New York,1951)

[8] Koshiba, M., Suzuki,M.: 'Finite-element analysis of H-plane waveguide junction with arbitrarily shaped ferrite post', IEEE Trans. Microw. Theory Tech., 1986, 34, (1), pp. 103-109

[9] Ise, K., Koshiba, M.: 'Equivalent circuits for dielectric posts in a rectangular waveguide', IEEE Trans. Microw. Theory Tech.,1989, 37, (11), pp. 1823-1825

[10] Ise, K., Koshiba, M.: 'Dielectric post resonances in a rectangular waveguide', IEE Proc., 1990, 137, (1), pp. 6166

[11] Auda, H. A., Smith, C. E.: 'The rsonances method for evaluating the impedances of the equivalent network for dielectric posts in a rectangular waveguide', IEEE Trans. Microw. Theory Tech., 1990, 38, (11), pp. 1595-1601

[12] Bachiller, C., Esteban, H., Mata, H., Valdes, M., Boria, V., Belenguer, A., Morro, J.: 'Hybrid mode matching method for the efficient analysis of metal and dielectric rods in H-plane rectangular waveguide devices', IEEE Trans. Microw. Theory Techn., 2010, 58, (12), pp. 3634-3644

[13] Caballero, E. D., Esteban, H., Belenguer, A., Boria, V.: 'Efficient analysis of substrate integrated waveguide devices using hybrid mode matching between cylindrical and guided modes', IEEE Trans. Microw. Theory Techn., 2012, 60, (2), pp. 232- 243

[14] Nafe, A., Shamim, A.: 'An integrable SIW phase shifter in partially magnetized ferrite LTCC package', IEEE Trans. Microw. Theory Techn., 2015, 63, (7), pp. 2264-2274

[15] Ghaffar, F. A., Shamim, A.: 'A partially magnetized ferrite LTCC based SIW phase shifter for phased array applications', IEEE Trans. Magn. 2015, 51, (6)

[16] Ghiotto, A., Adhikari, S., Wu, K., 'Ferrite-loaded substrate integrated waveguide switch', IEEE Microw. Compon. Lett., 2012, 22, (3), pp.120-122

[17] Adhikari, S., Ghiotto, A., Hemour S., Wu, K.: "Tunable nonreciprocal ferrite loaded SIW phase shifter", IEEE MTT-S Int. Microw. Symp. Dig., Jun. 2013. p.p. 1-3

[18] C. Balanis, Advanced Engineering Electromagnetics. New York: Wiley, 1989 


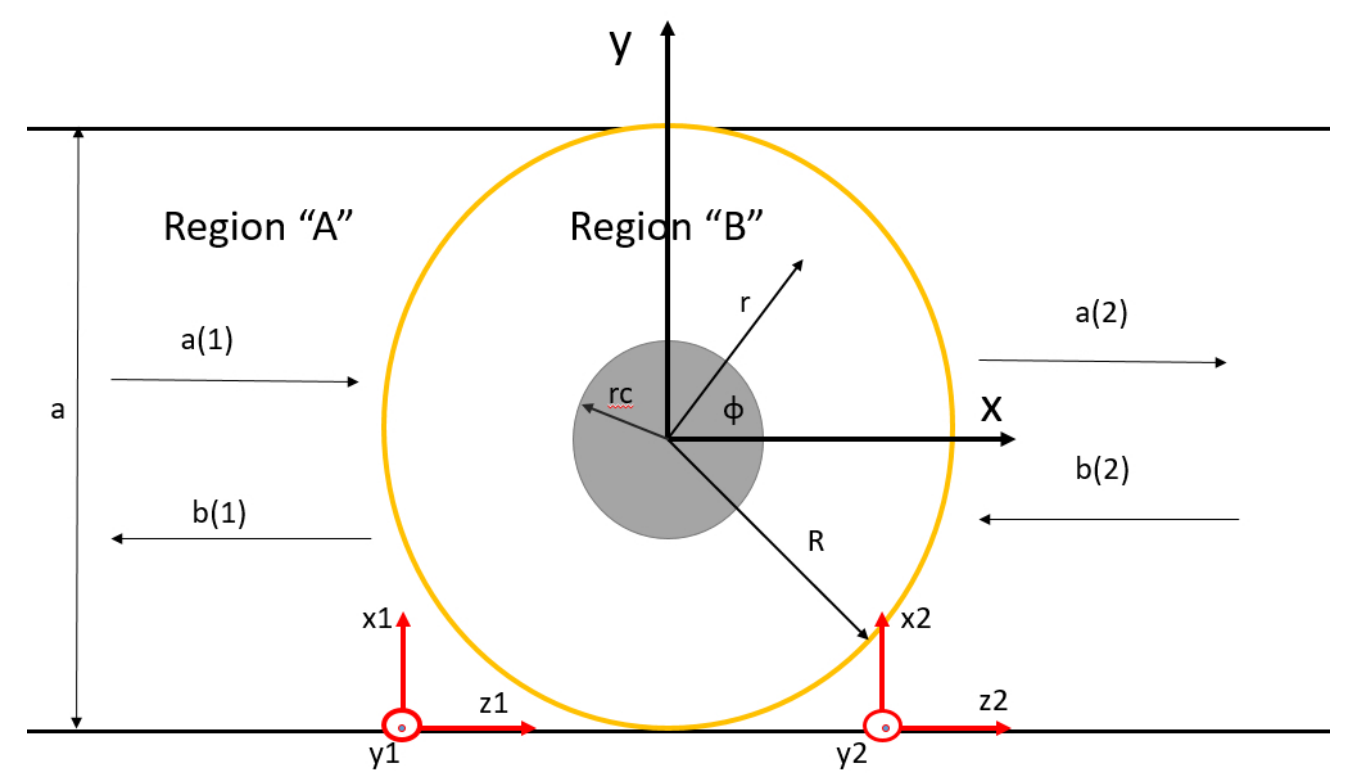

Fig. 1/ Problem Geometry $268 \times 165 \mathrm{~mm}(96 \times 96 \mathrm{DPI})$ 


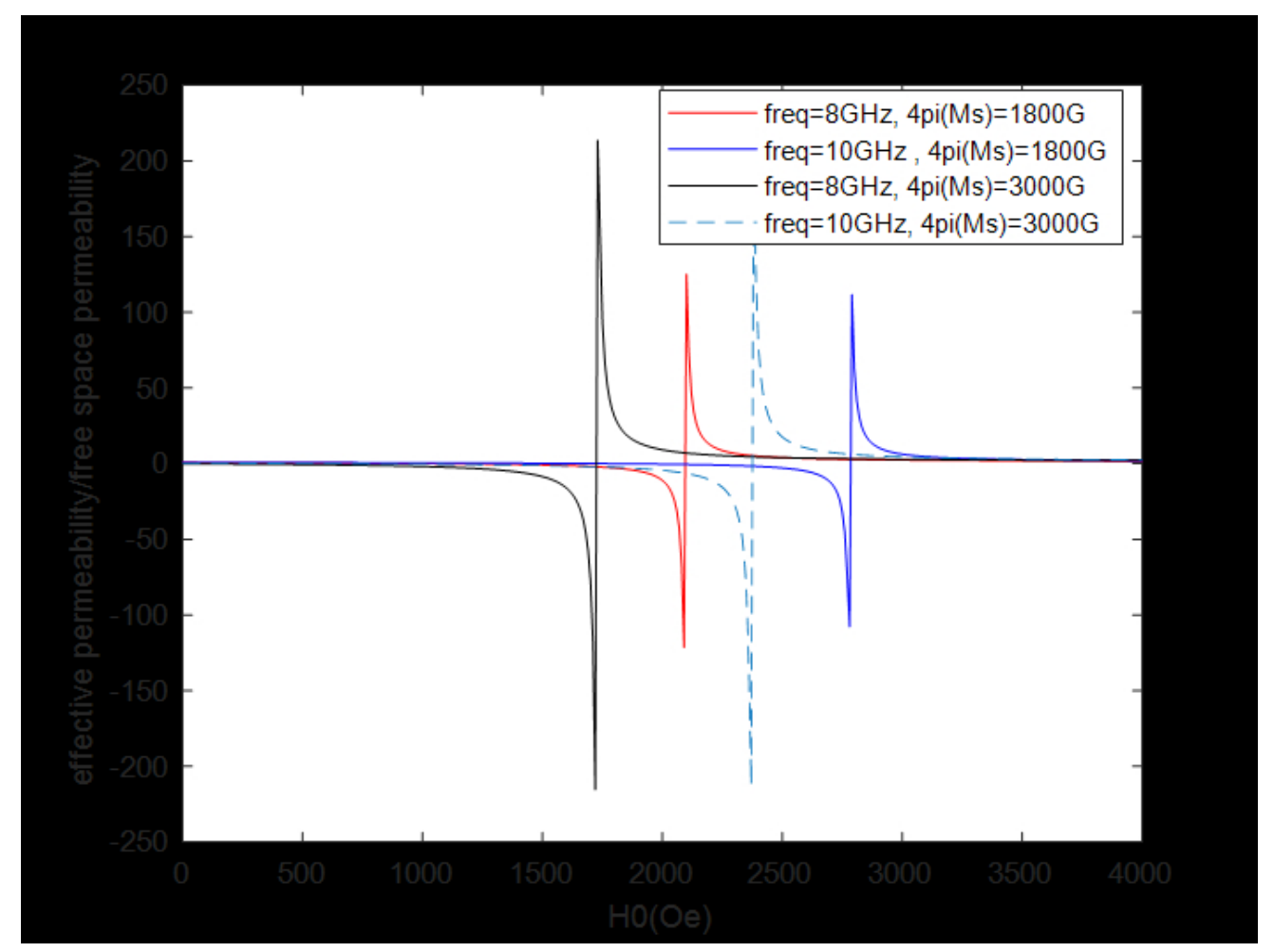

Fig. 2/Effective permeability for different frequency and magnetic bias $148 \times 111 \mathrm{~mm}(120 \times 120 \mathrm{DPI})$ 


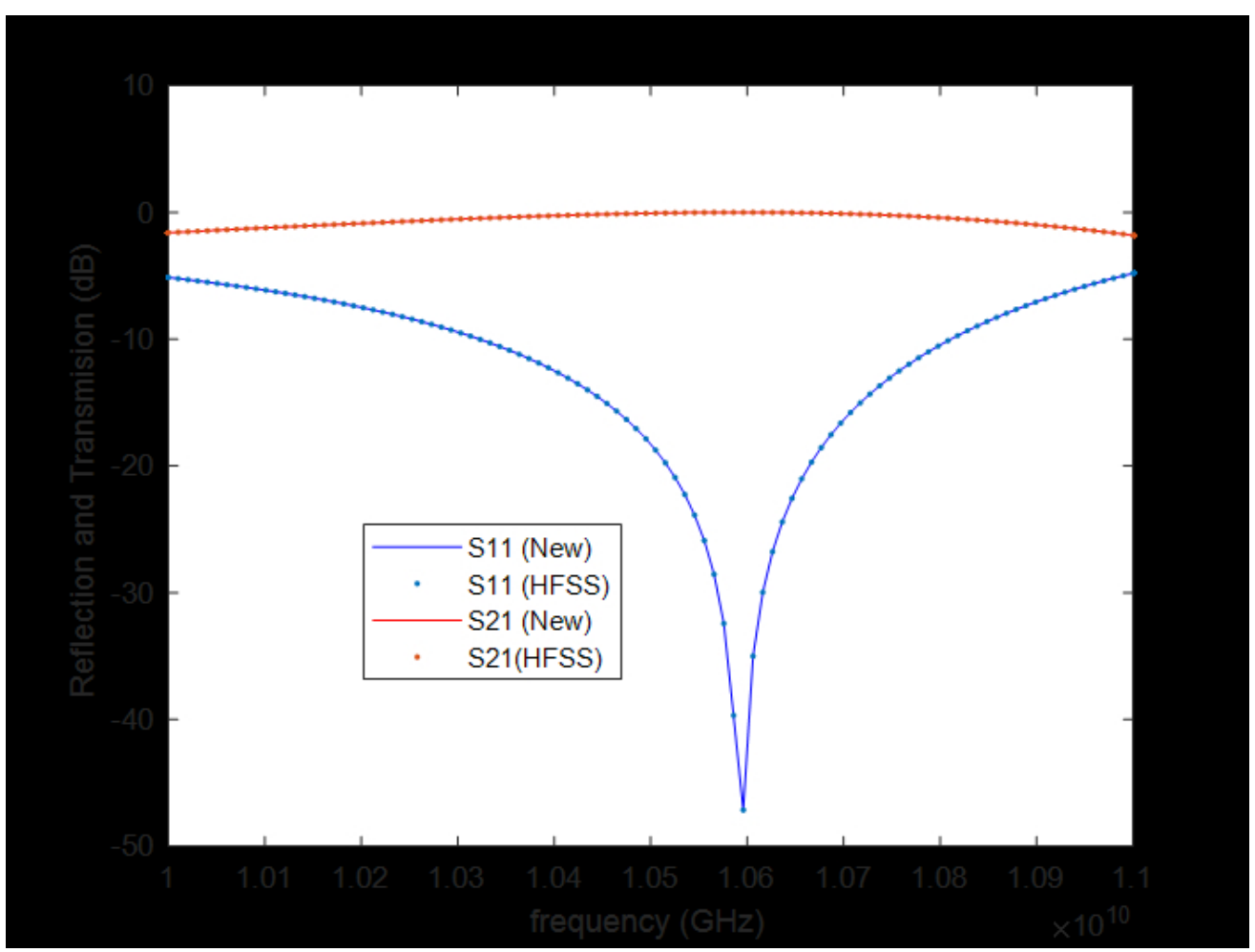

Fig. 3 / Single centered metal post. $a=7.112 \mathrm{~mm}, \mathrm{rc}=0.07 \mathrm{a}, \mathrm{HO}=3570 \mathrm{Oe}, \Delta \mathrm{H}=75 \mathrm{Oe}, \mathrm{Ms}=1800$ Gauss, ferrite permittivity constant $\varepsilon r f=13$.

$148 \times 111 \mathrm{~mm}(120 \times 120 \mathrm{DPI})$ 
Fig. 4/ Single centered dielectric post. $a=7.112 \mathrm{~mm}, \mathrm{rc}=0.07 \mathrm{a}, \mathrm{H} 0=3570 \mathrm{Oe}, \Delta \mathrm{H}=75 \mathrm{Oe}, \mathrm{Ms}=1800 \mathrm{Gauss}$, ferrite permittivity constant $\varepsilon r f=13$ and dielectric permittivity constant $\varepsilon r=24$.

\section{$148 \times 111 \mathrm{~mm}(120 \times 120 \mathrm{DPI})$}




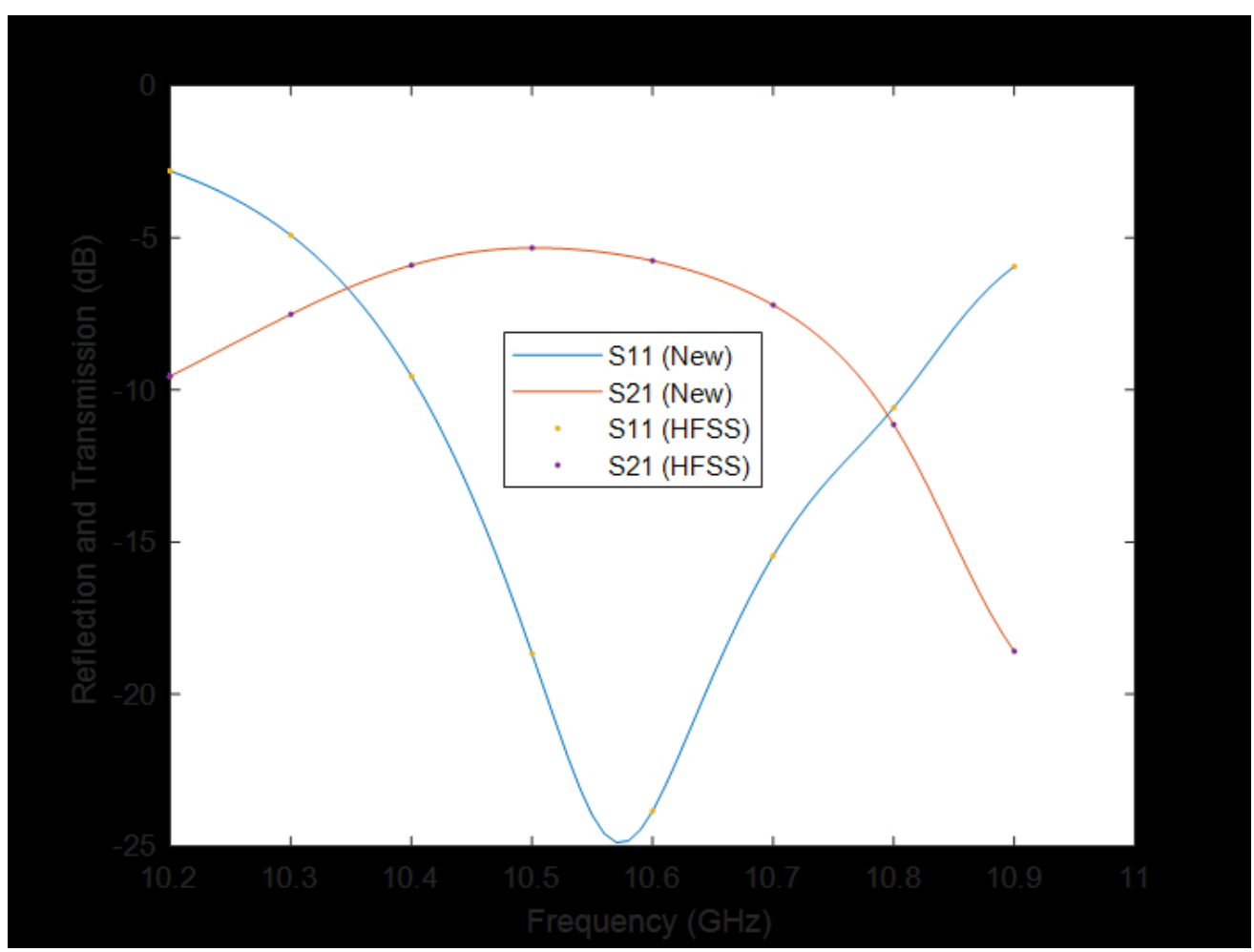

Fig. 5/ Three centered metal post. $\mathrm{a}=7.112 \mathrm{~mm}, \mathrm{rc}=0.07 \mathrm{a}, \mathrm{HO}=3570 \mathrm{Oe}, \Delta \mathrm{H}=75 \mathrm{Oe}, \mathrm{Ms}=1800$ Gauss, ferrite permittivity constant $\varepsilon r f=13$.

$148 \times 111 \mathrm{~mm}(120 \times 120 \mathrm{DPI})$ 